\title{
Microscopic theory of network glasses
}

\author{
Randall W. Hall \\ Department of Chemistry \\ Louisiana State University \\ Baton Rouge, La. 70803-1804 \\ Peter G. Wolynes \\ Department of Chemistry and Biochemistry \\ University of California, San Diego \\ La Jolla, Ca. 92093-0332
}

(Dated: November 1, 2018)

\begin{abstract}
A molecular theory of the glass transition of network forming liquids is developed using a combination of self-consistent phonon and liquid state approaches. Both the dynamical transition and the entropy crisis characteristic of random first order transitions are mapped out as a function of the degree of bonding and the density. Using a scaling relation for a soft-core model to crudely translate the densities into temperatures, the theory predicts that the ratio of the dynamical transition temperature to the laboratory transition temperature rises as the degree of bonding increases, while the Kauzmann temperature falls relative to the laboratory transition. These results indicate why highly coordinated liquids should be "strong" while van der Waals liquids without coordination are "fragile".
\end{abstract}

PACS numbers: 61.43.Fs,64.70.Pf,65.60.+a 
While much of the phenomenology of the glass transition is universal, shared by all dense liquids, everyday glasses are formed from liquids that possess a network structure at the molecular level. The universal features of the liquid-glass transition such as the Kauzmann entropy crisis and the Vogel-Fulcher rate law have been explained using theories based on an underlying random first-order transition [1, 2, 3], like that of mean field models with one step replica symmetry breaking [4]. Furthermore, the variations of the mean relaxation time 3$]$ and degree of nonexponentiality [5] of relaxation from substance to substance have been quantitatively predicted using this approach, provided the configurational heat capacity is known as input, thus quantitatively explaining the patterns of behavior verbally characterized using the expression "liquid fragility" [6]. Detailed microscopic calculations buttressing these developments are based on a model of a dense interacting atomic fluid in which the particles interact via van der Waals forces alone. Is this starting point appropriate for the network glasses? To address this issue, we explore the glass transition in a model of a network forming liquid. The calculations presented are similar in spirit to those made earlier for atomic fluids. Our model assumes cross-linking in a liquid is fluctuating, not quenched as in models of rubber [7], taking the mean degree of crosslinking as given from experiment or from a separate calculation explicitly accounting for the equilibria between the various different bonded species. These calculations suggest a connection between the glassy behavior of network systems as viewed by random first order transition theory and rigidity percolation. Rigidity percolation features prominently in Phillips's discussion of the role of bonding constraints in glass formation[8], as well as in the extensive studies of Thorpe [9, 10]. A major result of the present calculations is that the density of non-bonded interactions needed to trigger energy landscape dominated glassy behavior decreases when bonding increases. It follows that, consistent with experiment, highly cross-linked network glasses should show 
activated dynamics even at very high temperatures. The theory still predicts a crossover to non-activated dynamics for sufficiently high temperature as has recently been inferred in computer simulations of silica $[11,[12]$.

The self-consistent phonon method applied to the amorphous state provides our starting point 13, 14]. This scheme is closely connected to approaches based on density functionals [15]. It has recently been justified by an elegant clone or replica approach[16, 17]. We actually use a hybrid of both density functional and self-consistent phonon calculations. For the hard sphere system, both approaches agree there is a critical density $\rho_{A}$, below which localized density distributions corresponding to amorphous packings are mechanically unstable to even small thermal fluctuations. $\rho_{A} \sigma^{3}$ from the self-consistent theory is approximately 1.0 for hard spheres, where $\sigma$ is the hard sphere diameter. This is in agreement with the computer simulated dynamical transition[18]. This instability, from the vitrified side, coincides with the transition found in dynamical mode coupling approaches which start from the liquid side [1, 2, 19, 20]. The thermal vibration allowed at $\rho_{A}$ in a frozen amorphous state gives mean square fluctuations $\left\langle\delta r^{2}>=\alpha^{-1}\right.$, where $\alpha$ is an effective spring constant and is finite at the transition. $\alpha$ provides an analog of the Edwards-Anderson order parameter for spins and exhibits discontinuous replica symmetry breaking. This limit of stability for an amorphous system is comparable to the Lindemann criterion that describes melting of crystalline solids. At densities above $\rho_{A}$ many different mechanically stable amorphous packings or free energy minima can exist. At densities beyond $\rho_{A}$, however, the configurational entropy of these minima decreases until it vanishes at a density $\rho_{K}$. Analogously, for soft-sphere models at fixed densities one can find a temperature $T_{A}$, signaling the onset of glassy dynamics ("landscape determined" [21])and a still lower temperature $T_{K}$, the Kauzmann temperature where the configurational entropy would vanish. Between these 
densities or temperatures, the dynamics can be described using the concept of entropic droplets [1, 2, 3, 5]. The activation free energy diverges as $T_{K}$ is approached, consistent with the Vogel-Fulcher law $\Delta F^{\dagger}=D T_{K} /\left(T-T_{K}\right)$. A microscopic calculation of the energy cost of forming an entropic droplet quantitatively explains the experimentally observed variation of $\mathrm{D}$ with the heat capacity drop upon vitrification, $\Delta C_{p}[\underline{3}]$. This correlation relies on the idea that the Lindemann ratio near $T_{K}$ should depend only weakly on the detailed force law. We will check this supposition for our network glass model.

The free energy is computed for a given amorphous packing as a function of $\alpha$. This free energy should then be averaged over packings. Each atom can be assigned to a cell and the density can be written as $\rho(\mathbf{r})=\sum_{i} \rho_{i}=\sum_{i}\left(\frac{\alpha_{i}}{\pi}\right)^{3 / 2} \exp \left[-\alpha_{i}\left(\mathbf{r}-\mathbf{R}_{i}\right)^{2}\right]$ where the locations $\left\{\mathbf{R}_{i}\right\}$, characterize the packing as a set of fiducial locations around which the atoms vibrate. The free energy as a function of $\left\{\alpha_{i}\right\}, F\left(\left\{\alpha_{i}\right\}\right)$, is analogous to the inter replica potential given as a function of $\mathrm{q}$ for spin glasses [22].

More generally, the thermal vibrations are described by a tensor $\boldsymbol{\alpha}_{i}$ defined by

$$
\boldsymbol{\alpha}_{i}^{-1}=\left\langle\left(\mathbf{r}_{i}-\mathbf{R}_{i}\right)\left(\mathbf{r}_{i}-\mathbf{R}_{i}\right)\right\rangle_{W_{i}^{e f f}}
$$

where the averaging is with respect to the effective potential (dependent on $\left\{\alpha_{j}\right\}$ )

$$
\exp \left(-\beta W_{i}^{e f f}\right) \equiv \Pi_{i \neq j} \exp \left(-\beta V^{e f f}\left(\left|\mathbf{r}_{i}-\mathbf{R}_{j}\right|\right)\right)=\Pi_{i \neq j} \int \mathbf{d r}_{j} \rho_{j}\left(\mathbf{r}_{j}\right) \exp \left(-\frac{\beta}{2} V\left(r_{i j}\right)\right)
$$

where $V(r)$ is the potential energy. For an isotropic glass under the assumption that all $\alpha_{i}$ are the same $V^{e f f}$ becomes essentially a function of a scalar distance $\mathrm{R}, V^{e f f}(R)$, and one finds[14] $\alpha=\frac{\rho}{6} \int d R \quad g(R) \quad \nabla^{2}\left(\beta V^{\text {eff }}(R)\right)$ and a free energy $\frac{F(\alpha)}{N k_{B} T}=f^{\text {ideal }}+$ 
$\rho \quad \int d R g(R) V^{e f f}(R)$. Although a good approximation for large $\alpha$, the self-consistent phonon theory is not accurate for low $\alpha$. At low $\alpha$, an expansion around the uniform fluid can be used, but this depends on knowing the direct correlation function which is not available for network fluids. When no bonds are present, alternatively one can use the functional first suggested by Tarazona 23] and generalized by Ashcroft 24]. This functional takes the form $\frac{F}{N k_{b} T} \equiv f=\frac{1}{N} \int d r \rho(r)[\log (\rho(r))-1]+\Psi(\eta)$ where $\Psi$ is the interaction part of the free energy for a monatomic liquid or glass (which we take to be the Carnahan-Starling expression) and $\eta$ is the packing fraction $\left(\pi \rho \sigma^{3} / 6\right)$. For the network forming liquids we will correct this free energy using a modification of the result of Erukhimovich [25], which provides for the entropy loss of forming bonds.

For the network forming liquid and glass, we separate the Hamiltonian into bonding and non-bonding parts $V=\sum_{i, j}$ bonded $V_{b}\left(r_{i j}\right)+\sum_{i, j \text { non-bonded }} V_{n b}\left(r_{i j}\right)$. Each pair i and $\mathrm{j}$ is considered to be either bonded on non-bonded and thus have an interaction described by either $V_{b}$ or $V_{n b}$. The molecular units which are considered to be bonded have a locally harmonic potential energy $V_{b}\left(r_{i j}\right)=V_{0}+\frac{1}{2} \gamma^{b}\left(r_{i j}-d_{b}\right)^{2}$ with $d_{b}$ the equilibrium bond distance. $V_{n b}$, on the other hand, represents non-bonded interactions and therefore is taken to be a hard or soft sphere. Notice this potential does not explicitly describe, for any given configuration, whether a specific given pair should be considered bonded or not. This requires writing the potential using manybody switching functions [26, 27]. These manybody functions would reflect the saturation of chemical bonding tendencies (and would be needed for simulations.) For such a model the theory of associated liquids yields a (renormalized) equilibrium constant for the bonding reactions in terms of the underlying potential [28]. We will take the fraction of bonded pairs to be given and to be temperature and density independent. Of course, density and temperature dependent changes of the association equilibrium would be easy 
to include, but would distract from understanding how the glassy physics itself depends on temperature. Provided the association equilibrium constants are not paradoxically related to the density (i.e., decrease partially with $\rho$ ) the trends in any event will be as shown.

For the network system, the self-consistent phonon calculation must distinguish the two types of interactions. For specificity we assume any given atom can form 0-4 bonds, in a tetrahedral pattern. Other spatial patterns are easily treated as well. At large $\alpha$ the effective potential coming from a bonded pair is essentially $V_{b}^{e f f}(\mathbf{R})=\frac{V_{0}}{2}+\frac{\gamma^{b}}{4}\left(|\mathbf{R}|-d_{b}\right)^{2}$ where the factor of $1 / 2$ comes from the self-consistent formulation. We set the bond distance $d_{b}$ equal to the hard sphere diameter for the non-bonded atoms and choose a zero of energy such that $V_{0}=0$. In numerical calculations we used $\beta \gamma^{b}=300$, approximately the value appropriate for silica potentials [29]. The probability that a given atom has n-bonds, $p_{n}$ is given by Erukhimovich [25] $p_{n}=\left(\begin{array}{l}4 \\ n\end{array}\right) \Gamma^{n}(1-\Gamma)^{4-n}$ with $\Gamma=\frac{n_{b}}{4}$ and $n_{b}$ is the average number of bonds per atom.

In our calculations the basic pair distribution for non-bonded interactions, $g(R)$ is taken to be the Percus-Yevick radial distribution function modified for high density in the standard way. To account for the presence of bonded interactions that block the approach of other partners, $g(R)$ for an atom with $n$ bonds (denoted $\left.g_{n}(R)\right)$ is scaled by $\max \left(0, \frac{\langle n n\rangle_{\rho}-n}{<n n>_{\rho}}\right)$ for distances less than the first minimum of $\mathrm{g}(\mathrm{R}) .\langle n n\rangle_{\rho}$ is the number of nearest neighbors at total density $\rho$, obtained by integrating the first peak of $\mathrm{g}(\mathrm{R})$.

To each of the 5 types of atoms, those with $0,1,2,3$, or 4 bonds, we assign a mean $\alpha_{n}$. In this paper we use the uncoupled oscillator implementation of the self-consistent phonon theory 14] to determine the free energy. Essentially, this approximation treats the glass as a collection of Einstein oscillators. The derivation in Stoessel and Wolynes's early paper is lengthy in detail but when applied to the present potential one obtains 


$$
\begin{aligned}
F_{s c p} / N k_{B} T= & \sum_{n=0}^{4} p_{n}\left[V_{b, n}^{e f f}\left(d_{b}\right)+\rho \int d R g_{n}(R) V_{n b}^{e f f}(R)\right] \\
& -\ln \int_{v} d r^{N} e^{-\sum_{n=0}^{4} \frac{p_{n}}{2} r_{i} \cdot\left[\nabla \nabla \beta V_{b, n}^{e f f}\left(R_{i}\right)+\mathbf{I} \alpha_{n}^{n b}\right] \cdot r_{i}}
\end{aligned}
$$

where $V_{b, n}^{\text {eff }}$ is the sum of $V_{b}^{\text {eff }}$ for an atom with $n$ bonds over its bonds (and includes correlations between bonds through the use of a tetrahedral lattice), $V_{n b}^{\text {eff }}$ is defined by Eqn. 2] using only the non-bonding potential energy and $g_{n}$, and $\alpha_{n}^{n b}$ is the non-bonding contribution to $\alpha_{n}$. With a proper choice of local axes for each $r_{i}$, the argument of the exponential can be diagonalized, leading to 3 principal values of the vibrational tensor $\alpha_{1, n}$, $\alpha_{2, n}$, and $\alpha_{3, n}$. For the free energy to be consistent with Eqn. 11 we must have $\alpha_{n}$ satisfy $3 \alpha_{n}^{-1}=\alpha_{1, n}^{-1}+\alpha_{2, n}^{-1}+\alpha_{3, n}^{-1}$ Note that for a glass transition to occur, all 3 components of $\alpha_{n}$ must be large. Given a bonding pattern, the $\{\alpha\}$ can be determined and, hence, the free energy.

$F_{s c p}$ is the free energy of the large $\alpha$ glassy minimum. We compute the free energy of the network liquid with $\alpha=0$ using the Carnahan-Starling expression for a non-bonded liquid plus a modification of Erukhimovich's result for equilibrium network materials [25]: $F_{l i q} / N k_{B} T=\Psi(\eta)+4[\Gamma \ln \Gamma+(1-\Gamma) \ln (1-\Gamma)]+\ln \left(\frac{\rho}{e}\right)-\frac{n_{b}}{2} \ln \left(\frac{n_{b} \rho}{e}\right)+\frac{F_{s c p}^{b}}{N k_{B} T}$ where $F_{s c p}^{b}$ is obtained from Eqn. 3 with $\alpha=0$ and only the bonding terms. It corresponds to the entropy loss from bonding computed by Erukhimovich [25].

The self-consistent phonon theory alone allows us to find $\rho_{A}$, as the lowest density giving a non-zero $\alpha$, which thus corresponds to the onset of activated behavior while $\rho_{K}$, the "Kauzmann" density at which the free energies of the liquid and glass match and the configurational entropy vanishes also relies on the bonding correction to $F(\alpha=0)$. The ratio 
$\rho_{A} / \rho_{K}$ dimensionlessly characterizes the thermodynamic aspects completely. To compare to the kinetic laboratory glass transition, we note the laboratory glass transition defined to occur when the viscosity reaches $10^{14}$ p. Previous work using random first order theory predicts this to occur when the configurational entropy is about $1.0 k_{B}$. To make the translation of our thermodynamic results to the laboratory transition density in what follows we will therefore mean by $\rho_{G}$ the density where the liquid and glass free energies differ by 1.0 $k_{B} T$ per particle. The universality of configurational entropy at the laboratory transition is well confirmed, also by experiment.

$\rho_{A}, \rho_{K}$, and $\rho_{G}$ as functions of the average number of bonds $n_{b}$ are shown in Fig. 1 For $n_{b}>3, \rho_{A}$ vanishes, indicating that the rigidity percolation occurs when $n_{b}=3$ in our model. For comparison, Thorpe more precisely defining rigidity percolation as the absence of zero mode vibrational frequencies, finds $n_{b}=2.4$. For $n_{b}>3$, even at low densities the network is a rubber that can still vitrify at a higher densities. Fig. [2] shows the effective spring constants at these densities. For the hard sphere model[14] the uncoupled oscillator approximation leads to a somewhat lower $\rho_{A}$ than simulation and to relatively small, but nevertheless finite, values of $\alpha_{A}$. Quantitatively, the more complete coupled oscillator approximation (which uses a chain summation to treat the glass as a collection of Debye oscillators [14]) leads to a larger $\rho_{A}$ in better agreement with computer studies. So we would expect quantitative modification by taking better account of the coupling but expect the same trend, namely that as the amount of bonding is increased, the transition densities decrease and therefore the transition temperatures increase. This theory then explains why the addition of a non-bonding impurity into a networked glass will lower the glass transition temperature, as known since ancient times for silica $[30]$. In physical terms, the non-bonding transition densities decrease because, as seen in Eqn. 3 as the contribution 
from bonding to the vibrational force increases, the remaining contribution needed from non-bonding interactions for self-consistency decreases.

It is clear that $\rho_{A}$ has a much sharper dependence on density than either $\rho_{K}$ or $\rho_{G}$. The degree of bonding has a very direct effect on the vibrational force constant, being proportional to the average Laplacian of the effective potential, but only an indirect effect on entropy, entering through the more slowly varying (in space) Boltzmann factor. The theory explains the change of fragility with bonding. To see this we note that real liquids have both repulsive cores of finite strength and attractions which conspire in setting the equation of state. Yet assuming the attractions provide merely a smooth background potential the density dependences of this model can be translated into temperature dependences. For a soft potential $V \sim r^{-n}$ we have the scaling relation $T_{1} / T_{2}=\left(\rho_{2} / \rho_{1}\right)^{3 / n}$. Using this relation for $n=12$ yields $T_{A} / T_{G}$ and $T_{K} / T_{G}$ as plotted in Fig. 3. Experimental measurements of $T_{K} / T_{G}$ give 0.82 for the fragile glass orthoterphenyl [31], while for the strong glass silica the ratio has recently been determined[32] to be 0.36 . The uncoupled oscillator approximation results in a variation from 0.82 for the non-networked glass to 0.6 at the approximate rigidity percolation limit, a variation somewhat smaller in magnitude. Doubtless, the crudeness of this result is due to our use of a scaling approximation appropriate to a simple, purely repulsive model and better agreement with experiment would be obtained by including attractive forces in our estimates. We see that $T_{A} / T_{G}$ increases dramatically with $n_{b}$, while $T_{K} / T_{G}$ decreases modestly. Thus highly bonded liquids have large temperature ranges of nearly Arrhenius activated behavior while liquids with few crosslinks have smaller ranges of strongly non-Arrhenius activated behavior.

For the uncoupled oscillator treatment $\alpha_{A}$ falls rapidly with density to very small values as crosslinking increases. The universality of the Lindemann ratio previously assumed by 
Wolynes and coworkers [3] would seem in doubt. Yet this is not so. First, we know the coupled oscillator approximation will give a larger $\rho_{A}$ therefore decreasing the range of variation of $\alpha_{A}$. For $n_{b}=0$, the coupled oscillator gives a value of $\alpha_{A} \approx 100$, while the uncoupled theory gives $\alpha_{A} \approx 20[14]$. More important, even at the uncoupled level $\alpha_{K}$ and $\alpha_{G}$ are roughly constant with increasing bonding. A careful examination of the earlier analysis shows that these are the appropriate Lindemann-like ratios to determine the activation barriers in the strongly glassy regime. The predicted universal relation between $\mathrm{D}$ and $\Delta C_{p}$, relying on a universal $\alpha_{G}$, remains therefore sound.

The combination of self-consistent phonon and liquid state approaches provides a microscopic picture of network glasses that explains the dependence of the characteristic glass densities and, less directly, temperatures on degree of crosslinking and this results agrees with experiment. Doubtless, many of the detailed liquid state approximations made here can be improved but it is unlikely such modifications will change the qualitative story. In particular, the attractive forces that go beyond the soft-sphere model should probably be included to achieve quantitative agreement with experiment. This model combined with a theory of the association equilibria in forming the network provides a first principles treatment of glass properties starting from the underlying intermolecular forces.

RWH acknowledges NSF Grant. No. 9977124 for support.

[1] P.G.Wolynes, Acc. Chem. Res. 25, 513 (1992).

[2] T.R.Kirkpatrick and D.Thirumalai, Transp. Theor. Stat.Phys. 24, 927 (1995).

[3] X. Xia and P.G.Wolynes, PNAS 97, 2990 (2000).

[4] D.J.Gross, I.Kanter, and H.Sompolinsky, Phys. Rev. Lett. 55, 304 (1985). 


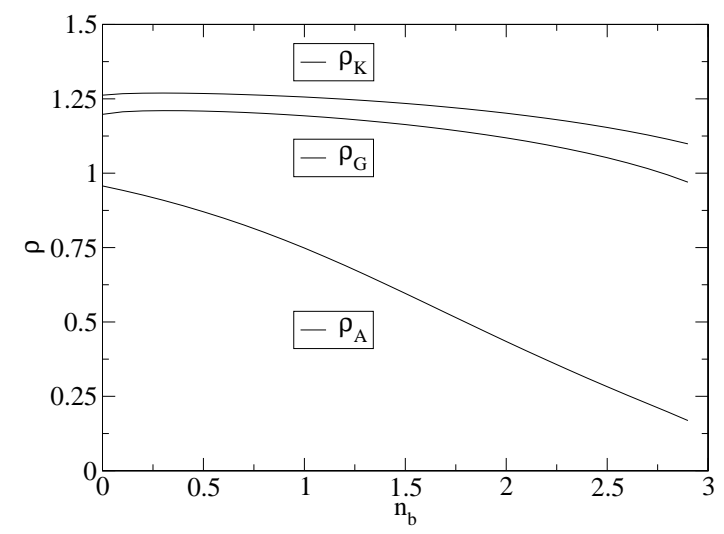

FIG. 1: Plot of $\rho_{A}, \rho_{K}$, and $\rho_{G}$ versus $n_{b}$.

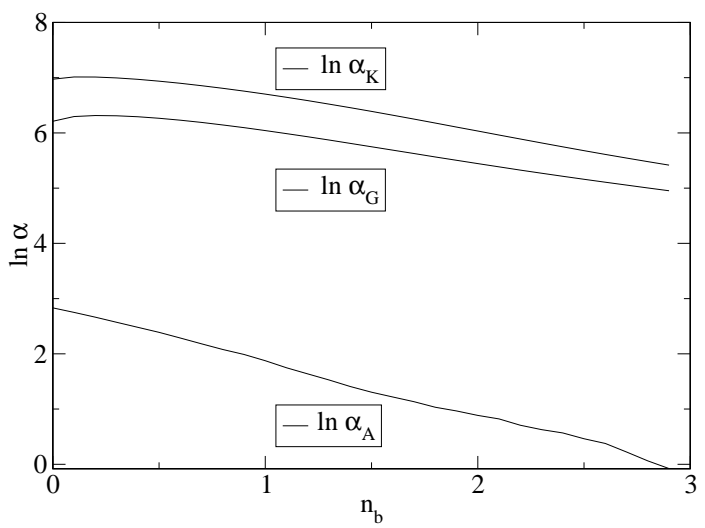

FIG. 2: Plot of $\ln \alpha_{A}, \ln \alpha_{K}$, and $\ln \alpha_{G}$ versus $n_{b}$.

[5] X. Xia and P.G.Wolynes, Phys. Rev. Lett. 86, 5526 (2001).

[6] C.A.Angell, J. Non-Crystalline solids 73, 1 (1985).

[7] P. Goldbart and N. Goldenfeld, Phys. Rev. A 39, 1402 (1989).

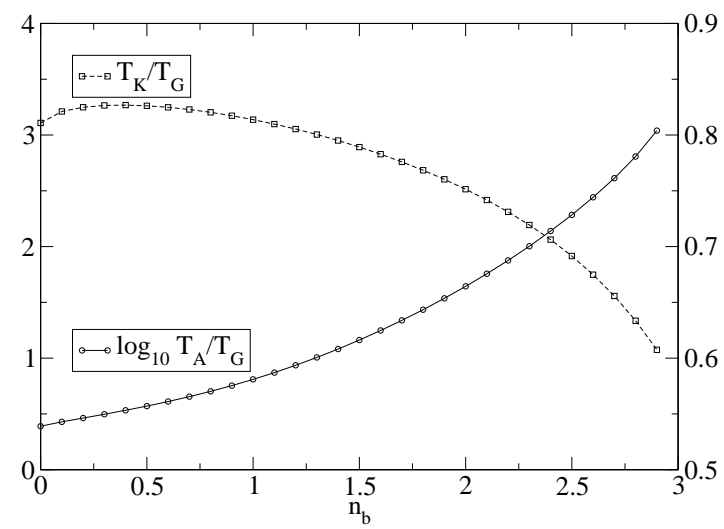

FIG. 3: Plot of $\log _{10}\left(T_{A} / T_{G}\right)$ (scale shown on left axis) and $T_{K} / T_{G}$ (scale shown on right axis) versus $n_{b}$. 
[8] J.C.Phillips, Solid State Commun. 53 (1985).

[9] S.Feng and M.F.Thorpe, Phys. Rev. B 31, 276 (1985).

[10] P.M.Duxbury, D.J.Jacobs, and M.F.Thorpe, Phys. Rev. E 59, 2084 (1999).

[11] J. Horbach and W. Kob, Phys. Rev. E 64, 041503 (2001).

[12] B. Coluzzi and P. Verrocchio, J. Chem. Phys. 116, 3789 (2002).

[13] M. Fixman, J. Chem. Phys. 51, 3270 (1969).

[14] J.P.Stoessel and P.G.Wolynes, J. Chem. Phys. 80, 4502 (1984).

[15] Y.Singh, J.P.Stoessel, and P.G.Wolynes, Phys. Rev. Lett. 54, 1059 (1985).

[16] S. Franz, M. Mezard, G. Parisi, and L. Peliti, J. Stat. Phys. 97, 459 (1999).

[17] M. Mezard and G. Parisi, 82, 747 (1999).

[18] L. Woodcock and C. Angell, Phys. Rev. Lett. 47, 1129 (1981).

[19] W. Götze, Condens. Matter 16, 873 (1999).

[20] U. Bengtzilius, W. Götze, and A. Sjölander, J. Phys. C 17, 5915 (1984).

[21] S.Sastry, P.G.Debenedetti, and F.H.Stillinger, Nature 393, 554 (1998).

[22] S.Franz and G.Parisi, Phys. Rev. Lett. 79, 2486 (1997).

[23] P.Tarazona, Molecular Physics 52, 81 (1984).

[24] A.Khein and N.W.Ashcroft, Phys. Rev. E 60, 2875 (1999).

[25] I. Erukhimovich, M. Thamm, and A. Ermoshkin, Macromolecules 34, 5653 (2001).

[26] F.H.Stillinger and T.A.Weber, Phys. Rev. B 31, 5262 (1985).

[27] L.R.Corrales and P.J.Rossky, Chem. Phys. Lett. 194, 363 (1992).

[28] L.W.Dahl and H.C.Andersen, J. Chem. Phys. 78, 1962 (1983).

[29] B. van Beest, G. Kramer, and R. van Santen, Phys. Rev. Lett. 64, 1955 (1990).

[30] Pliny the Elder, Natural History (xxxvi, 65). 
[31] C. Angell, J. Res. NIST 102, 171 (1997).

[32] A. Sipp, Y. Bottinga, and P. Richet, J. Non-Crystalline solids 288, 166 (2001). 